\title{
Coherent Excitation of the Highly Forbidden Electric Octupole Transition in ${ }^{172} \mathbf{Y b}^{+}$
}

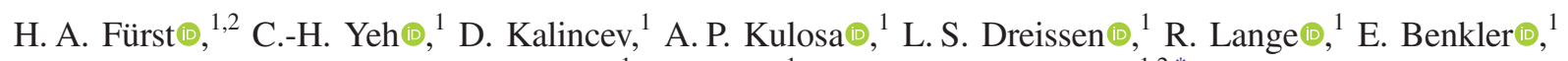 \\ N. Huntemann, ${ }^{1}$ E. Peik $\odot{ }^{1}{ }^{1}$ and T. E. Mehlstäubler ${ }^{1,2, *}$ \\ ${ }^{1}$ Physikalisch-Technische Bundesanstalt, Bundesallee 100, 38116 Braunschweig, Germany \\ ${ }^{2}$ Institut für Quantenoptik, Leibniz Universität Hanover, Welfengarten 1, 30167 Hanover, Germany
}

(Received 24 June 2020; accepted 28 August 2020; published 16 October 2020)

\begin{abstract}
We report on the first coherent excitation of the highly forbidden ${ }^{2} S_{1 / 2} \rightarrow{ }^{2} F_{7 / 2}$ electric octupole (E3) transition in a single trapped ${ }^{172} \mathrm{Yb}^{+}$ion, an isotope without nuclear spin. Using the transition in ${ }^{171} \mathrm{Yb}^{+}$as a reference, we determine the transition frequency to be $642116784950887.6(2.4) \mathrm{Hz}$. We map out the magnetic field environment using the forbidden ${ }^{2} S_{1 / 2} \rightarrow{ }^{2} D_{5 / 2}$ electric quadrupole (E2) transition and determine its frequency to be $729476867027206.8(4.4) \mathrm{Hz}$. Our results are a factor of $1 \times 10^{5}\left(3 \times 10^{5}\right)$ more accurate for the E2 (E3) transition compared to previous measurements. The results open up the way to search for new physics via precise isotope shift measurements and improved tests of local Lorentz invariance using the metastable ${ }^{2} F_{7 / 2}$ state of $\mathrm{Yb}^{+}$.

DOI: $10.1103 /$ PhysRevLett.125.163001
\end{abstract}

Introduction.-The standard model of particle physics successfully describes many phenomena of modern physics. However, it cannot be a complete description of nature as it does not explain experimental evidence of, e.g., dark matter and the matter-antimatter asymmetry within the universe. Moreover, gravitation, as covered by the welltested theory of general relativity, could not, up until now, be included in the standard model in a renormalizable way. Thus, tests of fundamental physics have become an important interdisciplinary field to gather new insights. Here, tabletop low energy atomic physics experiments profit from high precision spectroscopy to make them competitive compared to high energy experiments in the search for new physics [1]. In particular, the $\mathrm{Yb}^{+}$ion is an excellent candidate for this, as it features a directly accessible electric octupole (E3) transition with $\mathrm{nHz}$ linewidth to the electronic $F$ state, enabling the study of violations of local Lorentz invariance (LLI) with the highest sensitivity among accessible trapped ion systems [2-4], similar to the sensitivity of $\mathrm{Tm}$ in neutral atom systems [5]. In addition, the $\mathrm{Yb}^{+}$ion allows for straightforward laser cooling and has two narrow electric quadrupole (E2) transitions that can be accessed from the electronic ground state.

Access to seven stable isotopes of $\mathrm{Yb}^{+}$enables the search for new physics via the measurement of the isotope shifts (IS) of the three narrow optical transitions and their

Published by the American Physical Society under the terms of the Creative Commons Attribution 4.0 International license. Further distribution of this work must maintain attribution to the author(s) and the published article's title, journal citation, and DOI. analysis in King plots. These measurements are especially sensitive to a possible neutron number dependent fifth force, mediated by an unknown boson, coupling electrons with neutrons [6-9]. Very recent measurements of the E2 transitions in $\mathrm{Ca}^{+}$with accuracies on the order of $10 \mathrm{~Hz}$ $[10,11]$ have not yet led to significant signatures. Here, the $\mathrm{Yb}^{+}$ion is more suitable due to its higher sensitivity of a factor of 10 [8]. In fact, a deviation of $3 \sigma$ from an expected linear behavior of the King plot of the two E2 transitions ${ }^{2} S_{1 / 2} \rightarrow{ }^{2} D_{(3 / 2,5 / 2)}$ in even isotopes of $\mathrm{Yb}^{+}$was found recently [12]. The reported uncertainties of $\sim 300 \mathrm{~Hz}$ are not sufficient yet to attribute the deviation clearly to new physics, in contrast to higher-order contributions from nuclear structure [13]. More accurate measurements with uncertainties on the $\mathrm{Hz}$ down to $\mathrm{mHz}$ level, comparing the IS of the E2 and E3 transitions in $\mathrm{Yb}^{+}$, will provide a higher sensitivity to new physics as they are of different electronic types [8] and the uncertainties of the isotope mass can be eliminated using three transitions [9]. However, the required precision in the $\mathrm{Hz}$ range has not been realized so far in even isotopes of $\mathrm{Yb}^{+}$as their energy levels are magnetic field sensitive to the 1 st order. With this Letter, we open up the even isotope ${ }^{172} \mathrm{Yb}^{+}$for clock spectroscopy and demonstrate frequency uncertainties at the $\mathrm{Hz}$ level for both the ${ }^{2} S_{1 / 2} \rightarrow{ }^{2} D_{5 / 2}$ (E2) and the ${ }^{2} S_{1 / 2} \rightarrow{ }^{2} F_{7 / 2}$ (E3) transition and by this improve the literature values by at least 5 orders of magnitude $[14,15]$.

The first laser excitation of the highly forbidden E3 transition in ${ }^{172} \mathrm{Yb}^{+}$ions was carried out at the National Physical Laboratory and led to an uncertainty of $0.7 \mathrm{MHz}$ in the transition frequency and an excitation rate of about $0.03 \mathrm{~s}^{-1}$ on resonance [14]. Here, we demonstrate the first coherent quantum state control by achieving a $90 \%$ 
(a)

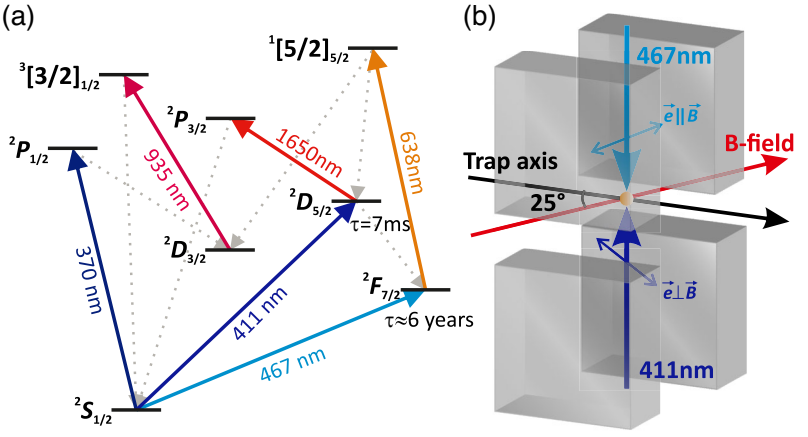

FIG. 1. Relevant optical transitions and laser access. (a) Reduced level scheme of ${ }^{172} \mathrm{Yb}^{+}$. Doppler cooling and repumping is carried out on the transitions near $370 \mathrm{~nm}$ and $935 \mathrm{~nm}$, respectively. Optical pumping into the $m_{J}$ electronic ground states is done via a $\sigma$-polarized $370 \mathrm{~nm}$ beam. Excitation of the metastable $D_{5 / 2}$ and $F_{7 / 2}$ states via the transition near $411 \mathrm{~nm}$ and $467 \mathrm{~nm}$ are detected using fluorescence imaging on the cooling transition, followed by repumping using the transitions near $1650 \mathrm{~nm}$ and $638 \mathrm{~nm}$. (b) Laser access and $\vec{B}$-field orientation for the probe beams of polarization $\vec{e}$ within the ion trap segment (gray).

excitation probability for Rabi spectroscopy with $42 \mathrm{~ms}$ long pulses. The coherence time is found to be 190(27) ms, and the achieved minimum linewidth of the transition is 6.0(6) $\mathrm{Hz}$. By referencing our probe laser to the E3 clock transition in ${ }^{171} \mathrm{Yb}^{+}\left(F=0 \rightarrow 3, \Delta m_{F}=0\right)$, we derive the E3 transition frequency in ${ }^{172} \mathrm{Yb}^{+}$with an uncertainty of $2.4 \mathrm{~Hz}$. For the E2 transition frequency, we obtain an uncertainty of $4.4 \mathrm{~Hz}$. For both frequency measurements, we discuss the uncertainties and show that they can be kept at sub-Hz level for transition frequency differences between different isotopes. Our results will open up a way to precise IS measurements in $\mathrm{Yb}^{+}$and for a rapid and defined preparation of the $F_{7 / 2}$ state for sensitive tests of LLI, increasing current limits by 2 orders of magnitude $[3,4]$.

Experimental setup.-Our experiment is carried out in an rf Paul trap, as described in Ref. [16]. Single ions are Doppler cooled to $0.5(1) \mathrm{mK}$ on the transition near $370 \mathrm{~nm}$, assisted by a repumper laser near $935 \mathrm{~nm}$ (see Fig. 1). For the interrogation of the E2 transition, we use a frequencydoubled diode laser near $822 \mathrm{~nm}$, locked to a cavity with a fractional instability of $5 \times 10^{-16}$ at $10 \mathrm{~s}$ averaging time [17], providing the short-term stability of the system. The light is amplified with an injection-locked laser diode and the frequency doubled in a periodically poled potassium titanyl phosphate crystal to $411 \mathrm{~nm}$. A maximum power of about $0.6 \mathrm{~mW}$ is focused down to a waist of $83 \mu \mathrm{m}$ at the position of the ion.

Coherent excitation of the E3 transition requires an ultrastable, high intensity laser source near $467 \mathrm{~nm}$. For that we use a seed laser power of about $0.5 \mathrm{~mW}$ near $934 \mathrm{~nm}$ from the probe laser of the ${ }^{171} \mathrm{Yb}^{+}$single ion optical clock [18] via a stabilized fiber link. We use an acoustooptical modulator near $2.3 \mathrm{GHz}$ to bridge the frequency difference and two injection-locked laser diodes for light amplification. The light is frequency doubled in a periodically poled $\mathrm{LiNbO}_{3}$ waveguide to $467 \mathrm{~nm}$. We obtain about $8 \mathrm{~mW}$ of probe light with beam waists of $\left(w_{x}, w_{y}\right)=$ $[26(3), 38(3)] \mu \mathrm{m}$ at the ion. During the spectroscopic interrogation, the $934 \mathrm{~nm}$ laser is referenced to the E3 clock transition of ${ }^{171} \mathrm{Yb}^{+}$, a recommended secondary representation of the Système International second $[19,20]$.

For both probe lasers, power stabilization, switching, and frequency tuning are performed via acousto-optical modulators. Spectroscopy is carried out after optical pumping using circularly polarized cooling light to prepare the population in one of the $m_{J}= \pm 1 / 2$ electronic ground states, followed by the respective probe laser pulse. Excitation is detected by the absence of fluorescence at the $370 \mathrm{~nm}$ cooling transition (electron shelving). Repumping is carried out as shown in Fig. 1.

Results on the ${ }^{2} S_{1 / 2} \rightarrow{ }^{2} D_{5 / 2}$ transition.-By coherent controlling the E2 transition near $411 \mathrm{~nm}$, we achieve an excitation of over $95 \%$ for a $\pi$ pulse of $t_{\pi}=8.6 \mu \mathrm{s}$ duration. Using this transition, we map out and minimize magnetic field noise at the ion position by actively stabilizing the magnetic field as described in [21]. Further, we align the focus of the $467 \mathrm{~nm}$ spectroscopy beam onto the ion by maximizing the induced ac Stark shift on the E2 $\left(m_{J}=-1 / 2 \rightarrow-5 / 2\right)$ transition. A frequency shift of $2.1(1) \mathrm{kHz}$ at a peak intensity of $I_{467}=5.2(7) \times$ $10^{6} \mathrm{~W} / \mathrm{m}^{2}$ is observed for a polarization of the $467 \mathrm{~nm}$ beam parallel to the quantization axis [see Fig. 1(b)].

We obtain spectra of the two $m_{J}= \pm 1 / 2 \rightarrow \pm 5 / 2$ $\left(\Delta m_{J}= \pm 2\right)$ transitions by measuring the population in the $D_{5 / 2}$ state as a function of the frequency of the excitation laser. Alternating the transitions cancels out slow drifts in the magnetic field. We use excitation pulses of $t_{\pi}=1.6 \mathrm{~ms}$ that lead to about an $80 \%$ excitation probability. Averaging 40 spectra measured within $1.5 \mathrm{~h}$ leads to a statistical uncertainty of $2.5 \mathrm{~Hz}$. During this period, the $822 \mathrm{~nm}$ master laser frequency is calibrated using the primary frequency standard CSF2 [27] via an optical frequency comb $[28,29]$ that uses a hydrogen maser as a flywheel oscillator. A drift of the $822 \mathrm{~nm}$ locking cavity was accounted for via the data obtained by the frequency comb measurement. The short averaging time leads to an additional statistical uncertainty of $1.3 \mathrm{~Hz}$. We determine the center frequency of the E2 transition to be $729476867027206.8(4.4) \mathrm{Hz}$, improving on the uncertainty given in the literature by a factor of $1.0 \times 10^{5}$ [15]. The frequency is corrected for known systematic shifts, as discussed at the end of this Letter.

Excitation of the ${ }^{2} S_{1 / 2} \rightarrow{ }^{2} F_{7 / 2}$ transition.-To initially observe the E3 transition within the large uncertainty interval of $1.4 \mathrm{MHz}$ [14], we employ a rapid adiabatic passage (RAP) technique. The technique allows for a robust excitation of a transition in the presence of noise, e.g., from the laser source or magnetic fields [30]. This is 
achieved by sweeping the laser frequency adiabatically across the resonance during the spectroscopy pulse. For a reasonably slow sweep rate $\alpha \ll \Omega \cdot \Gamma$ (with $\Gamma<\Omega$ ), the transition probability reaches at least $50 \%$ without exact knowledge of the present noise figure $\Gamma$ and origin (e.g., frequency fluctuations of the probe laser) and exact resonant Rabi frequency $\Omega[30,31]$. We choose the $\Delta m_{J}=0$ transitions as they are the least magnetic field sensitive $( \pm 6 \mathrm{kHz} / \mu \mathrm{T})$ in a field of $6.5 \mu \mathrm{T}$. After minimization of environmental noise sources, we use pulses of maximum intensity and sweep the detuning across the expected resonance in windows of $\Delta f_{\mathrm{RAP}}=200 \mathrm{~Hz}$ for a pulse length of $t_{\mathrm{RAP}}=1 \mathrm{~s}$. We optimize the excitation probability $P_{F_{7 / 2}}$ by varying the sweep rate $\alpha=\Delta f_{\mathrm{RAP}} /$ $t_{\text {RAP }}$ as shown in blue in Fig. 2(a). The data (black) is fitted using the model in Refs. [30,31],

$$
P_{F_{7 / 2}}=\left(1-e^{-\Omega^{2} / 4 \alpha}\right) e^{-\Gamma \Omega / 2 \alpha}+\frac{1}{2}\left(1-e^{-\Gamma \Omega / 2 \alpha}\right) .
$$

The fit leads to $\Omega /(2 \pi)=9.6(5) \mathrm{Hz}$ and a noise figure of $\Gamma /(2 \pi)=3.0(9) \mathrm{Hz}$. At this Rabi frequency, a maximum excitation probability of $P_{F_{7 / 2}}=60 \%$ is obtained for pulse times of $360 \mathrm{~ms}(\alpha=556 \mathrm{~Hz} / \mathrm{s})$, limited by the noise figure $\Gamma$ being similar to $\Omega$. However, the RAP technique can serve as a helpful tool to efficiently find the transition in other isotopes when looking for anomalies in IS for the search of new physics due to a potential neutron number dependent fifth force $[8,12]$.

To achieve a higher spectroscopic resolution and faster population transfer, as required for an efficient test of LLI in the $F$ manifold of $\mathrm{Yb}^{+}$, we use Rabi spectroscopy with pulses of constant frequency and intensity. Within the $200 \mathrm{~Hz}$ window identified with RAP, the resonance can be found easily. On resonance, Rabi spectroscopy at maximum power leads to $P_{F_{7 / 2}}=90.0(1) \%$, as depicted in blue in Fig. 2(b), where Rabi oscillations of the $F$ state population are shown along with a sinusoidal fit (black) with exponential decaying envelope. The fit leads to decoherence time of $\tau=190(27) \mathrm{ms}$ and $\Omega /(2 \pi)=11.90(14) \mathrm{Hz}$, similar to the RAP model. Note that achieving a similar excitation probability using the RAP method would require an intensity of a factor 180(20) higher [Fig. 2(a), solid red] to satisfy $\Omega \gg \Gamma$.

To investigate the minimum achievable linewidth of the transition, we varied the intensity of the spectroscopy beam and the resonant $\pi$ pulse time accordingly. The fitted linewidths of the spectra (FWHM) for several pulse times are shown in Fig. 3 (blue). The black line corresponds to the Fourier limit of $\Delta f_{\pi} \approx 0.89 / t_{\pi}$ for rectangular shaped $\pi$ pulses. The inset shows a spectrum for $t_{467}=265 \mathrm{~ms}$, deviating from the Fourier limit by additional 2.5(7) Hz due to incoherent contributions of the magnetic field during the $180 \mathrm{~s}$ of measurement time. The additional broadening is in
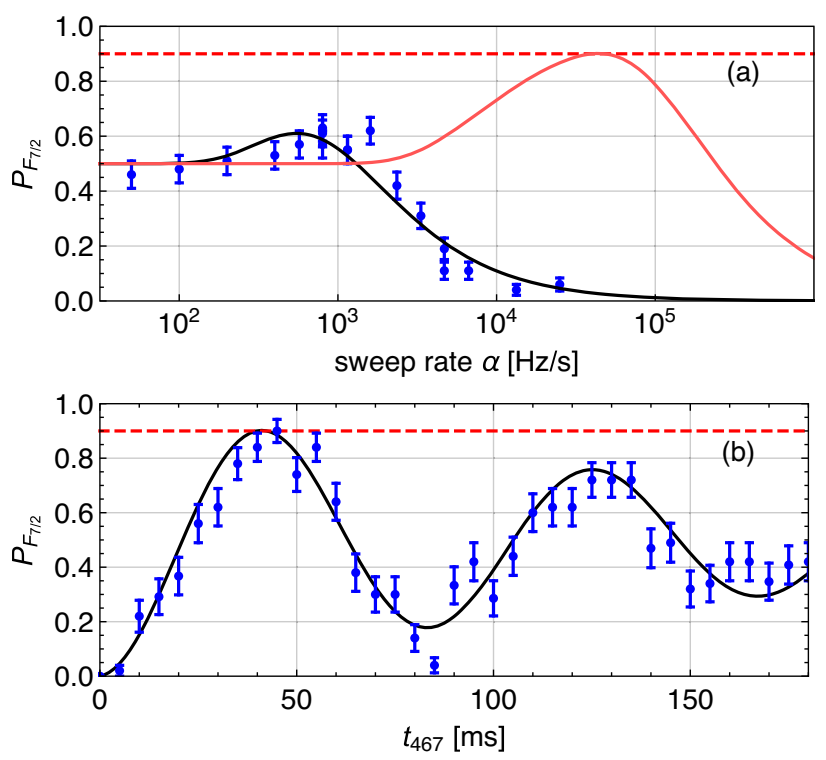

FIG. 2. Laser excitation of the $F$ state. (a) Excitation probability for a RAP pulse covering the resonance within a window of $200 \mathrm{~Hz}$ vs sweep rate $\alpha$ (blue) and a fit according to Eq. (1) (black). (b) Rabi flop on the $|S,-1 / 2\rangle \rightarrow|F,-1 / 2\rangle$ E3 transition (blue) along with a sinusoidal fit with exponential envelope (black), leading to a Rabi frequency of $11.90(14) \mathrm{Hz}$ and a decoherence time of $\tau=190(27) \mathrm{ms}$. Each point was averaged over 50 measurements. In both plots the red dashed line indicates a $90 \%$ excitation probability. This required a Rabi frequency of $130 \mathrm{~Hz}$ when using a RAP instead [(a), solid red].

agreement with the noise level extracted from the RAP measurements.

To determine the frequency, we alternately measure the spectra of the two $\Delta m_{J}=0$ Zeeman components using $\pi$ pulses of $124 \mathrm{~ms}$ to achieve 1st order magnetic field insensitivity. We average the data over 16 spectra measured within $1 \mathrm{~h}$, leading to a statistical uncertainty of $0.13 \mathrm{~Hz}$. We determine the frequency shift to the $F=0 \rightarrow F=3$

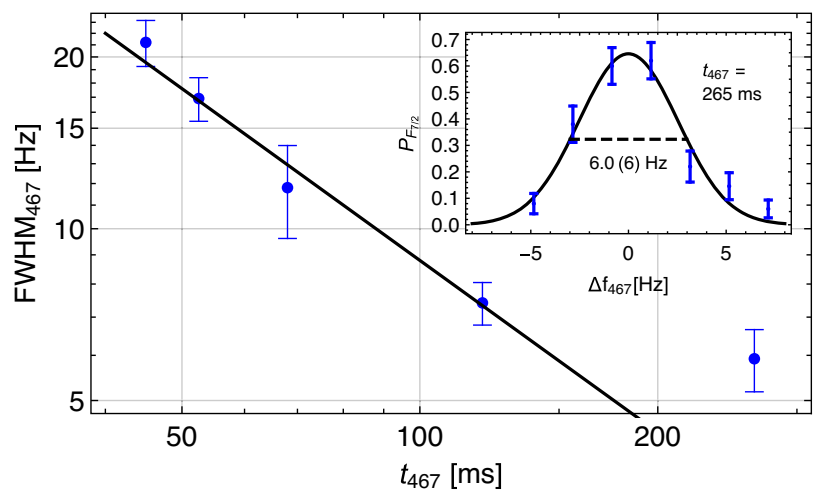

FIG. 3. Observed transition linewidth FWHM 467 vs length $t_{467}$ of the spectroscopy pulse (blue) along with the Fourier limited linewidth for the used pulse shape (black). The inset shows the spectrum at $t_{467}=265 \mathrm{~ms}$ (blue) along with a Gaussian fit (black). 
TABLE I. Leading systematic frequency shifts $\delta \nu$ and related uncertainties $\mu$ in $\mathrm{Hz}$ for the ${ }^{2} S_{1 / 2} \rightarrow{ }^{2} D_{5 / 2}$ quadrupole (E2) and ${ }^{2} S_{1 / 2} \rightarrow{ }^{2} F_{7 / 2}$ octupole (E3) transition in ${ }^{171} \mathrm{Yb}^{+}$.

\begin{tabular}{lcccccc}
\hline \hline & \multicolumn{2}{c}{$\mathrm{E} 2$} & & \multicolumn{2}{c}{$\mathrm{E} 3$} \\
\cline { 2 - 3 } \cline { 6 - 7 } Effect & $\delta \nu$ & $\mu$ & & $\delta \nu$ & $\mu$ \\
\hline 935 nm ac Stark & 8.8 & 2.7 & & $\ldots$ \\
Quadrupole shift & -9.9 & 2.0 & & -0.07 & 0.01 \\
Stark shift (probe light) & 0.003 & 0.002 & & 33.0 & 2.3 \\
Blackbody radiation & -0.24 & 0.11 & & -0.07 & 0.03 \\
Total & -1.3 & 3.4 & & 32.9 & 2.3 \\
\hline \hline
\end{tabular}

$\left(\Delta m_{F}=0\right)$ clock transition to be $f_{E 3}^{(172)}-f_{E 3}^{(171)}=$ $-4711821757.3(2.3) \mathrm{Hz}$.

For the determination of the transition frequency, the uncertainty of the recommended value of the reference frequency in ${ }^{171} \mathrm{Yb}^{+}$of $0.39 \mathrm{~Hz}$ [20] is taken into account. This yields $f_{E 3}^{(172)}=642116784950887.6(2.4) \mathrm{Hz}$. The frequencies are corrected for known systematic shifts as discussed in the following.

Systematic shifts and uncertainties.-Major contributions to the systematic shifts and their uncertainties are summarized in Table I.

For both transitions, an electric quadrupole shift resulting from the dc trapping potential [32] and stray electric fields has to be considered. The shift is calculated to be $-9.9(2.0) \mathrm{Hz}$ for the E2 transition and -0.07 (1) $\mathrm{Hz}$ for the E3 transition for an axial trap frequency of 213(5) $\mathrm{kHz}$ and an angle of $25(5)^{\circ}$ between the trap axis and the magnetic field. The quadrupole moments are taken from Refs. [33,34], respectively. The effect of stray electric fields can be estimated from the dc fields required to compensate excess micromotion and is at least an order of magnitude smaller and is included in the uncertainty budget. Note that our trap frequencies, and thus field gradients, show a long-term stability of below $10^{-4}$, and quadrupole shifts can be measured and monitored at the $\mathrm{mHz}$ level if needed [35].

The large intensity of the probe light leads to a significant ac Stark shift of the E3 transition. We deduce the shift by measuring the resonance frequency at different optical powers and extrapolate to zero as described in [21]. With this, we obtain an ac Stark shift of 33.0(2.3) Hz for the $124 \mathrm{~ms} \pi$ pulses used in the frequency measurement. For the E2 transition, the ac Stark shift is much smaller. Using the information provided by Refs. [36-39], we compute the differential polarizability of the used $m_{J}= \pm 1 / 2 \rightarrow \pm 5 / 2$ transition at $411 \mathrm{~nm}$ to be $2.8(1.5) \times 10^{-39} \mathrm{Jm}^{2} \mathrm{~V}^{-2}$, leading to a shift of $0.003(2) \mathrm{Hz}$ for the used $\pi$ pulses of $1.6 \mathrm{~ms}$.

An additional Stark shift stems from blackbody radiation (BBR) [40]. At an effective temperature at the ion position of 297(3) K [41,42], the BBR shift is calculated to be
$-0.24(11) \mathrm{Hz}$ for the E2 and $-0.07(3) \mathrm{Hz}$ for the E3 transition, limited by the instability of the lab temperature. The involved differential polarizability for the E2 transition is computed to be $-4.5(2.3) \times 10^{-40} \mathrm{Jm}^{2} \mathrm{~V}^{-2}$ using Refs. [36-39], where the static value of $-4.3(2.2) \times$ $10^{-40} \mathrm{Jm}^{2} \mathrm{~V}^{-2}$ was corrected for the BBR spectrum $[38,43]$, whereas for the E3 transition the value can be found in Ref. [19]. Due to the lack of a fast laser shutter during the frequency measurement, the $935 \mathrm{~nm}$ repumper beam was present during the $\mathrm{E} 2$ interrogation, leading to an ac Stark shift. We determine the shift to be $8.8(2.7) \mathrm{Hz}$ in a separate measurement with interleaved interrogation of the E2 transition with and without the $935 \mathrm{~nm}$ laser field. Additional systematic shifts $<0.02 \mathrm{~Hz}$ are discussed in the Supplemental Material [21].

Combining statistical and systematic uncertainties, we obtain a total uncertainty of $4.4 \mathrm{~Hz}$ and $2.4 \mathrm{~Hz}$ for the frequencies of the E2 and E3 transitions, respectively.

Conclusion.-We report on the first coherent excitation of the highly forbidden E3 transition in the even isotope ${ }^{171} \mathrm{Yb}^{+}$with an excitation probability of $90 \%$ within $42 \mathrm{~ms}$. Fast and reliable state preparation in the $F_{7 / 2}$ state Zeeman manifold is an important requirement for an improved test of LLI with well-controllable trapped ion Coulomb crystals of ${ }^{171} \mathrm{Yb}^{+}[4,40]$ to enable a high duty cycle and give a high signal-to-noise ratio for the LLI signal.

We measured the frequencies of the E2 transition near $411 \mathrm{~nm}$ and the E3 transition with an uncertainty of $4.4 \mathrm{~Hz}$ and $2.4 \mathrm{~Hz}$, respectively. In particular, in Ref. [8] it was proposed that reaching the $\mathrm{Hz}$ level in uncertainties of the isotope shifts (IS) of these transitions should allow one to investigate the so-called ${ }^{8} \mathrm{Be}$ anomaly [44].

Probing the sub- $\mathrm{Hz}$ regime in IS for the E3 and both E2 transitions is predicted to provide further insights, as the measurement of a third transition will eliminate mass uncertainties and higher-order standard model contributions $[9,45]$, which can also lead to a nonlinearity $[13,46]$. Sub-Hz accuracy of the systematic shifts can be achieved by transferring techniques demonstrated in the ${ }^{171} \mathrm{Yb}^{+}$ clock spectroscopy $[18,47]$ to the even isotopes. Alternating interrogation of different isotopes suppresses common mode effects [12] such as the quadrupole shift, BBR shift, and trap-rf-related Stark shift, as the large uncertainties of the quadrupole moments and of the polarizabilities drop out. The BBR shifts can be kept reproducible at the $\mathrm{mHz}$ level if the temperature is stabilized to $\Delta T<0.3 \mathrm{~K}$. For the quadrupole shift, the influence of the uncertainties of electric field gradient and angle of quantization axis can be kept stable at sub-mHz levels [35]. Alternatively, entanglement of different cotrapped isotopes as presented in Ref. [48] can be applied to $\mathrm{Yb}^{+}$and has proven to reach $\mathrm{mHz}$ accuracies in IS measurements of $\mathrm{Sr}^{+}$.

We kindly acknowledge help from Stefan Weyers in the frequency measurement of the quadrupole transition and 
Atish Roy for support with computation of the $D_{5 / 2}$ state polarizabilities. We thank Michel Wolf and Tjeerd J. Pinkert for work on the magnetic field stabilization and Michael Drewsen for fruitful discussions. This project has been supported by the Deutsche Forschungsgemeinschaft (DFG, German Research Foundation) through Grant No. CRC SFB 1227 (DQ-mat, project B03) and through Germany's Excellence Strategy EXC-2123 QuantumFrontiers-390837967. This work has been supported by the EMPIR Project No. 18SIB05 "Robust Optical Clocks for International Timescales." This project has received funding from the EMPIR program cofinanced by the Participating States and from the European Unions Horizon 2020 research and innovation program. This work has been supported by the Max-Planck-RIKEN-PTB-Center for Time, Constants and Fundamental Symmetries.

*Corresponding author tanja.mehlstaeubler@ptb.de

[1] M. S. Safronova, D. Budker, D. DeMille, D. F. Jackson Kimball, A. Derevianko, and C. W. Clark, Rev. Mod. Phys. 90, 025008 (2018).

[2] V. Dzuba, V. Flambaum, M. Safronova, S. Porsev, T. Pruttivarasin, M. Hohensee, and H. Häffner, Nat. Phys. 12, 465 (2016).

[3] C. Sanner, N. Huntemann, R. Lange, C. Tamm, E. Peik, M. S. Safronova, and S. G. Porsev, Nature (London) 567, 204 (2019).

[4] R. Shaniv, R. Ozeri, M. S. Safronova, S. G. Porsev, V. A. Dzuba, V. V. Flambaum, and H. Häffner, Phys. Rev. Lett. 120, 103202 (2018).

[5] A. Golovizin, E. Fedorova, D. Tregubov, D. Sukachev, K. Khabarova, V. Sorokin, and N. Kolachevsky, Nat. Commun. 10, 1724 (2019).

[6] C. Delaunay, R. Ozeri, G. Perez, and Y. Soreq, Phys. Rev. D 96, 093001 (2017).

[7] V. V. Flambaum, A. J. Geddes, and A. V. Viatkina, Phys. Rev. A 97, 032510 (2018).

[8] J. C. Berengut, D. Budker, C. Delaunay, V. V. Flambaum, C. Frugiuele, E. Fuchs, C. Grojean, R. Harnik, R. Ozeri, G. Perez, and Y. Soreq, Phys. Rev. Lett. 120, 091801 (2018).

[9] J. C. Berengut, C. Delaunay, A. Geddes, and Y. Soreq, arXiv:2005.06144.

[10] F. W. Knollmann, A. N. Patel, and S. C. Doret, Phys. Rev. A 100, 022514 (2019).

[11] C. Solaro, S. Meyer, K. Fisher, J. C. Berengut, E. Fuchs, and M. Drewsen, arXiv:2005.00529 [Phys. Rev. Lett. (to be published)].

[12] I. Counts, J. Hur, D. P. L. A. Craik, H. Jeon, C. Leung, J. Berengut, A. Geddes, A. Kawasaki, W. Jhe, and V. Vuletić, arXiv:2004.11383 [Phys. Rev. Lett. (to be published)].

[13] S. O. Allehabi, V. A. Dzuba, V. V. Flambaum, A. V. Afanasjev, and S.E. Agbemava, Phys. Rev. C 102, 024326 (2020).

[14] M. Roberts, P. Taylor, G. P. Barwood, P. Gill, H. A. Klein, and W. R. C. Rowley, Phys. Rev. Lett. 78, 1876 (1997).
[15] P. Taylor, M. Roberts, S. V. Gateva-Kostova, R. B. M. Clarke, G. P. Barwood, W. R. C. Rowley, and P. Gill, Phys. Rev. A 56, 2699 (1997).

[16] J. Keller, D. Kalincev, T. Burgermeister, A. P. Kulosa, A. Didier, T. Nordmann, J. Kiethe, and T. E. Mehlstäubler, Phys. Rev. Applied 11, 011002 (2019).

[17] J. Keller, S. Ignatovich, S. A. Webster, and T. E. Mehlstäubler, Appl. Phys. B 116, 203 (2014).

[18] N. Huntemann, C. Sanner, B. Lipphardt, C. Tamm, and E. Peik, Phys. Rev. Lett. 116, 063001 (2016).

[19] N. Huntemann, M. Okhapkin, B. Lipphardt, S. Weyers, C. Tamm, and E. Peik, Phys. Rev. Lett. 108, 090801 (2012).

[20] F. Riehle, P. Gill, F. Arias, and L. Robertsson, Metrologia 55, 188 (2018).

[21] See Supplemental Material, which includes Refs. [22-26], at http://link.aps.org/supplemental/10.1103/PhysRevLett .125 .163001 for details about the magnetic field stabilization and systematic frequency shifts.

[22] B. Merkel, K. Thirumalai, J. E. Tarlton, V. M. Schäfer, C. J. Ballance, T. P. Harty, and D. M. Lucas, Rev. Sci. Instrum. 90, 044702 (2019).

[23] N. Herschbach, K. Pyka, J. Keller, and T. E. Mehlstäubler, Appl. Phys. B 107, 891 (2012).

[24] NIST Atomic Spectra Database http://physics.nist.gov/ PhysRefData/ASD.

[25] H. C. J. Gan, G. Maslennikov, K.-W. Tseng, T. R. Tan, R. Kaewuam, K. J. Arnold, D. Matsukevich, and M. D. Barrett, Phys. Rev. A 98, 032514 (2018).

[26] H. Denker, L. Timmen, C. Voigt, S. Weyers, E. Peik, H. S. Margolis, P. Delva, P. Wolf, and G. Petit, J. Geodes. 92, 487 (2018)

[27] S. Weyers, V. Gerginov, M. Kazda, J. Rahm, B. Lipphardt, G. Dobrev, and K. Gibble, Metrologia 55, 789 (2018).

[28] S. Falke, H. Schnatz, J. S. R. Vellore Winfred, T. Middelmann, S. Vogt, S. Weyers, B. Lipphardt, G. Grosche, F. Riehle, U. Sterr, and C. Lisdat, Metrologia 48, 399 (2011).

[29] R. Schwarz, S. Dörscher, A. Al-Masoudi, E. Benkler, T. Legero, U. Sterr, S. Weyers, J. Rahm, B. Lipphardt, and C. Lisdat, Phys. Rev. Research 2, 033242 (2020).

[30] T. Noel, M. R. Dietrich, N. Kurz, G. Shu, J. Wright, and B. B. Blinov, Phys. Rev. A 85, 023401 (2012).

[31] X. Lacour, S. Guérin, L. P. Yatsenko, N. V. Vitanov, and H. R. Jauslin, Phys. Rev. A 75, 033417 (2007).

[32] W. Itano, J. Res. Natl. Inst. Stand. Technol. 105, 829 (2000).

[33] D. K. Nandy and B. K. Sahoo, Phys. Rev. A 90, 050503(R) (2014).

[34] R. Lange, N. Huntemann, C. Sanner, H. Shao, B. Lipphardt, C. Tamm, and E. Peik, arXiv:2005.14687 [Phys. Rev. Lett. (to be published)].

[35] P. Dubé, A. A. Madej, J. E. Bernard, L. Marmet, J.-S. Boulanger, and S. Cundy, Phys. Rev. Lett. 95, 033001 (2005).

[36] T. Feldker, H. Fürst, N. V. Ewald, J. Joger, and R. Gerritsma, Phys. Rev. A 97, 032511 (2018).

[37] A. Roy, S. De, B. Arora, and B. Sahoo, J. Phys. B 50, 205201 (2017).

[38] T. Schneider, Ph.D. Thesis, University of Hanover, 2005.

[39] A. Roy (private communication). 
[40] J. Keller, T. Burgermeister, D. Kalincev, A. Didier, A. P. Kulosa, T. Nordmann, J. Kiethe, and T. E. Mehlstäubler, Phys. Rev. A 99, 013405 (2019).

[41] M. Doležal, P. Balling, P. B. R. Nisbet-Jones, S. A. King, J. M. Jones, H. A. Klein, P. Gill, T. Lindvall, A. E. Wallin, M. Merimaa, C. Tamm, C. Sanner, N. Huntemann, N. Scharnhorst, I. D. Leroux, P. O. Schmidt, T. Burgermeister, T.E. Mehlstäubler, and E. Peik, Metrologia 52, 842 (2015).

[42] T. Nordmann, A. Didier, M. Doležal, P. Balling, T. Burgermeister, and T. E. Mehlstäubler, arXiv:2008.04231.

[43] J. W. Farley and W. H. Wing, Phys. Rev. A 23, 2397 (1981).
[44] A. J. Krasznahorkay, M. Csatlós, L. Csige, Z. Gácsi, J. Gulyás, M. Hunyadi, I. Kuti, B. M. Nyakó, L. Stuhl, J. Timár, T. G. Tornyi, Z. Vajta, T. J. Ketel, and A. Krasznahorkay, Phys. Rev. Lett. 116, 042501 (2016).

[45] K. Mikami, M. Tanaka, and Y. Yamamoto, Eur. Phys. J. C 77, 896 (2017)

[46] M. Tanaka and Y. Yamamoto, arXiv:1911.05345.

[47] C. Tamm, N. Huntemann, B. Lipphardt, V. Gerginov, N. Nemitz, M. Kazda, S. Weyers, and E. Peik, Phys. Rev. A 89, 023820 (2014).

[48] T. Manovitz, R. Shaniv, Y. Shapira, R. Ozeri, and N. Akerman, Phys. Rev. Lett. 123, 203001 (2019). 